\title{
Reduced Measurement-space Dynamic State Estimation (ReMeDySE) for Power Systems
}

\author{
Jinghe Zhang, Student Member, IEEE, Greg Welch, Member, IEEE, \\ Gary Bishop and Zhenyu Huang Senior Member, IEEE
}

\begin{abstract}
Applying Kalman filtering techniques to dynamic state estimation is a developing research area in modern power systems. Compared to traditional steady state estimators, the Kalman filter is able to track dynamic state variables both efficiently and accurately. However, in large-scale and widearea interconnected power systems, the combination of computational complexity-primarily due to the very large number of measurements-and slow processing speeds present a significant challenge. To help address this challenge we have developed an approach we call Reduced Measurement-space Dynamic State Estimation (ReMeDySE). We present the method in the context of the Kalman filter, however it can also be applied to other state estimation methods such as particle filters. In addition, although we present the method in the context of power systems, it is suitable for real-time and massive calculations in any largescale state tracking systems. Finally, the method lends itself well to modern parallel computation techniques for further improvements.
\end{abstract}

Index Terms-Power systems, Dynamic State Estimation, Dynamic Measurement Selection, Power system simulation, Kalman Filter, Parallel Compuatation

\section{INTRODUCTION}

$\mathbf{S}$ TATE estimation is a fundamental and very important function in modern power system operations. It seeks to produce a reliable dynamic database for use in critical operational functions including real-time security monitoring, loadforecasting, economic despatch, and load-frequency control.

There are typically four steps involved in any state estimation procedure:

1) Modeling. In this step, network topology is determined, preliminary data are checked and calculated.

2) Observability determination. Various sensor measurements are classified as either critical or redundant. Critical measurements are necessary to achieve the system observability, so if some critical measurements are lost, pseudo measurements can be included. On the other hand, redundant measurements can be removed without affecting the system observability.

3) State estimation. Typically, a state estimator receives telemetered measurements from a Supervisory Control And Data Acquisition (SCADA) system in the time

This work supported by U.S. DOE grant DE-SC0002271, which is a subcontract from PNNL, PI Zhenyu "Henry" Huang. At DOE, grant managed by Sandy Landsberg, Program Manager for Applied Mathematics Research; Office of Advanced Scientific Computing Research; DOE Office of Science.

J. Zhang, G. Welch and G. Bishop are members of the Department of Computer Science, The University of North Carolina at Chapel Hill, Chapel Hill, NC, 27599-3175 USA e-mail: \{jing2009, welch, gb $@$ @ cs.unc.edu

Z. Huang is with Battelle-Pacific Northwest National Laboratory, Richland, WA 99352 USA (e-mail: zhenyu.huang@pnl.gov). interval of several seconds. From the measurements and an assumed steady state system model, the state estimator generates a set of static state variables that reflect a best estimate of the system conditions.

4) Detection and identification of gross measurement errors and/or errors in network structure.

Kalman filtering techniques have been applied to improve the computational performance of the traditional state estimation process in power system applications since the 1970's [1], [2]. However under the assumption of a steady state system model, none of the dynamic characteristics of a power system are reflected in the estimation. This has not been an issue historically as SCADA measurements are too infrequent to capture the system dynamics. This changed with the introduction of Phasor Measurement Unit (PMU). PMUs primarily make two types of measurements available: voltage and current phasors, from which line power flow and bus power injection can be derived. Recent developments of phasor measurement technologies provide high-speed sensor data (typically 30 samples/second) with precise time synchronization [3], [4], motivating the reexamination of the exclusion of dynamic states in the state estimation process. For example, in [5] we investigated the feasibility of applying Kalman filtering techniques to include dynamic state elements in the state estimation process, e.g., generator rotor angles and generator speeds, in addition to the static state elements of voltage magnitudes and phase angles. The study results present a promising path forward for the implementation of Kalman filter based dynamic state estimation in conjunction with the emerging phasor measurement technologies.

As a result of the increasing size and complexity of the interconnected power networks, the computational complexity of full state estimation remains an obstacle to be overcome. Even with modern supercomputers, the massive data processing is still a very challenging task given the time and space (memory) complexities. This is despite significant work aimed at accelerating the calculations via parallel implementations such as OpenMP [7] and Global Arrays [8].

Here we introduce a different approach to reduce the computational burden. Previously we introduced an estimation approach called single-constraint-at-a-time (SCAAT) [9]. The idea behind the SCAAT approach is to use a Kalman filter to estimate a globally observable system using low dimensional measurements from potentially unobservable subspaces. The benefits include higher sampling rates, lower latencies, and improved accuracy. The approach inspired us to design LoDiM [10], a SCAAT-based approach to state tracking for large-scale systems. In this paper, we present ReMeDySE, a lightweight 
yet efficient estimation approach to capture the dynamic states in power systems. Furthermore, our method is compatible with other powerful tools such as hierarchical estimation, distributed estimation, and parallel computation techniques.

The remainder of this paper is organized as follows. Section III presents the motivation of our approach with some discussion of historical circumstances and some related work. In , we formulate the power system dynamic state estimation problem, and present our new approach, which is aimed at dramatically reducing the computational requirements without sacrificing the tracking quality. In Section IV we present simulated results of tests on a multi-machine system model, to demonstrate how it could benefit the dynamic state estimation process. Finally we offer concluding thoughts in Section V.

\section{BACKGROUND AND RELATED WORK}

The phasor measurement unit (PMU) was first developed and utilized in [3], [4]. In [5] we investigated the feasibility of applying Kalman filtering techniques to include dynamic state variables in the estimation process. The results indicated a promising path forward for the implementation of Kalman filter based dynamic state estimation, in conjunction with the emerging PMU measurement technologies.

In applications such as power systems, computational load is always a concern. Significant previous work has attempted to reduce the computational complexity. For example, in [12] the authors proposed a measurement reduction approach for systems where there are more measurements than states. They described an equivalent state-space system can be used where the number of measurements equals the number of states. However, they assumed the system is static and linear, and did not take the preparation overhead into account.

In [13] the authors illustrated a measurement selection procedure for Extended Kalman filters. Nevertheless, as stated by the authors, an inherent limitation of the proposed method is that measurement selection is based entirely on the steadystate sensitivity matrix. However in this approach, the actual information content of the candidate measurements under typical operating conditions is not considered; the measurement rankings obtained are local and dependent on the steady state chosen as the base case; and dynamic and nonlinear effects are neglected.

Previously in [9], we presented SCAAT, a Kalman filterbased incremental tracking algorithm. It estimates a globally observable system using only measurements from locally unobservable systems. The underlying principle is that the single observations provide some information about the user's state, and thus can be used to incrementally improve a previous estimate. We then developed LoDiM with more sophisticated measurement selection procedure [10], such that the estimation has higher reporting rates and lower latency than the classic Kalman filter. Here we take the dynamic states of power systems into consideration and present ReMeDySE, a lightweight yet efficient estimator that is suitable for reflecting power system dynamic characteristics.

\section{Reduced Measurement-SPace Dynamic State ESTIMATION}

The ReMeDySE approach employs an estimator such as the Kalman filter, but with a twist: while the filter is running in the foreground, it also performs a measurement selection procedure in the background, where the selection is designed to optimally reduce the measurement dimension. As a result, during each cycle it yields much smaller computational load and hence lower latency. In this section, we first describe the relevant dynamic state space models, then provide a brief introduction to the Kalman filter (used to illustrate the approach), and then discuss the principles and structure of ReMeDySE.

\section{A. The Power System Dynamic State Space}

An assumed linear system can be modeled as a pair of linear stochastic process and measurement equations

$$
\begin{aligned}
x_{k} & =A x_{k-1}+w_{k-1} \\
z_{k} & =H x_{k}+v_{k}
\end{aligned}
$$

where $x \in \mathcal{R}^{n}$ is the state vector, $z \in \mathcal{R}^{m}$ is the measurement vector, $A$ is a $n \times n$ matrix that relates the state at the previous time step $k-1$ to the state at the current step $k$ in the absence of either a driving function or process noise ${ }^{1}$, and $H$ is an $m \times n$ matrix that relates the state $x$ to the measurement $z$. The process noise $w$ and measurement noise $v$ are assumed to be mutually independent random variables, spectrally white, and with normal probability distributions

$$
\begin{aligned}
p(w) & \sim N(0, Q) \\
p(v) & \sim N(0, R),
\end{aligned}
$$

where the process noise covariance $Q$ and measurement noise covariance $R$ matrices are often assumed to be constant.

In practice the process to be estimated and (or) the measurement relationship to the process are usually nonlinear, for example when the objective is to estimate the dynamic states of a power system. A nonlinear system can be modeled using nonlinear stochastic process and measurement equations

$$
\begin{aligned}
x_{k} & =a\left(x_{k-1}, w_{k-1}\right) \\
z_{k} & =h\left(x_{k}, v_{k}\right) .
\end{aligned}
$$

One can approximate the states and measurements by

$$
\begin{aligned}
x_{k} & =a\left(x_{k-1}\right) \\
z_{k} & =h\left(x_{k}\right) .
\end{aligned}
$$

These nonlinear functions can then be linearized about the point of interest $x$ in the state space. To do so one needs to compute either or both of the Jacobian matrices

$$
\begin{aligned}
& A=\left.\frac{\partial a(x)}{\partial x}\right|_{x} \\
& H=\left.\frac{\partial h(x)}{\partial x}\right|_{x}
\end{aligned}
$$

where $A$ and $H$ are the partial derivatives of $a$ and $h$ (respectively) with respect to the elements of the state $x$.

\footnotetext{
${ }^{1}$ In practice, the matrix $A$ may change with each time step
} 
The specific state elements we consider here are generator rotor angles and generator speeds. Without loss of generality, in a power system that consists of $n$ generators and $m$ buses, let us consider the generator $i$ which is connected to the generator terminal bus $i$. We use a classical model for the generator composed of a voltage source $\left|E_{i}\right| \angle \delta_{i}$ with constant amplitude behind an impedance $X_{d_{i}}^{\prime}$. The nonlinear differential-algebraic equations regarding generator $i$ can be written as

$$
\left\{\begin{aligned}
\frac{d \delta_{i}}{d t} & =\omega_{B}\left(\omega_{i}-\omega_{0}\right) \\
\frac{d \omega_{i}}{d t} & =\frac{\omega_{0}}{2 H_{i}}\left(P_{m_{i}}-\frac{\left|E_{i}\right|\left|V_{i}\right|}{X_{d_{i}}^{\prime}} \sin \left(\delta_{i}-\theta_{i}\right)-D_{i}\left(\omega_{i}-\omega_{0}\right)\right)
\end{aligned}\right.
$$

where state variables $\delta$ and $\omega$ are the generator rotor angle and speed respectively, $\omega_{B}$ and $\omega_{0}$ are the speed base and the synchronous speed in per unit, $P_{m_{i}}$ is the mechanical input, $H_{i}$ is the machine inertia ${ }^{2}, D_{i}$ is the generator damping coefficient and $\left|V_{i}\right| \angle \theta_{i}$ is the phasor voltage at generator terminal bus $i$.

For the state vector $x=\left[\delta_{1}, \omega_{1}, \delta_{2}, \omega_{2}, \ldots, \delta_{n}, \omega_{n}\right]^{T}$, the corresponding continuous time change in state can be modeled by the differential equation

$$
\frac{d x}{d t}=A_{c} x+w_{c}
$$

where $w_{c}$ is an $2 n \times 1$ continuous time process noise vector with $2 n \times 2 n$ noise covariance matrix $Q_{c}=E\left[w_{c} w_{c}^{T}\right]$, and $A_{c}$ is a $2 n \times 2 n$ continuous time state transition Jacobian matrix.

The detailed derivations of the corresponding discretetime state transition matrix $A$ and discrete-time process noise covariance $Q$ are described in [5] and [6]. According to the process model from equation (1) we have

$$
\begin{aligned}
x_{k} & =A x_{k-1}+w_{k-1} \\
& =\left(I+A_{c} \cdot \Delta t\right) x_{k-1}+w_{k-1},
\end{aligned}
$$

where $w$ is the process noise with normal probability distribution $p(w) \sim N(0, Q)$.

Power system measurments include bus voltages, bus angles and line flows. The measurement equations thus can be derived using the nodal admittance matrix and bus voltage reconstruction matrix, according to the expanded system nodal equation:

$$
Y_{\exp }\left(\begin{array}{c}
E \\
V
\end{array}\right)=\left(\begin{array}{ll}
Y_{G G} & Y_{G L} \\
Y_{L G} & Y_{L L}
\end{array}\right)\left(\begin{array}{c}
E \\
V
\end{array}\right)=\left(\begin{array}{c}
I_{G} \\
0
\end{array}\right)
$$

where $E=\left[\left|E_{1}\right| \angle \delta_{1},\left|E_{2}\right| \angle \delta_{2}, \ldots,\left|E_{n}\right| \angle \delta_{n}\right]^{T}$ is the vector of internal generator complex voltages, $V=$ $\left[\left|V_{1}\right| \angle \theta_{1},\left|V_{2}\right| \angle \theta_{2}, \ldots,\left|V_{n}\right| \angle \theta_{n}\right]^{T}$ is the vector of bus complex voltages, $I_{G}$ represents electrical currents injected by generators, $Y_{\text {exp }}$ is called the expanded nodal matrix, which includes loads and generator internal impedances: $Y_{G G}, Y_{G L}, Y_{L G}$ and $Y_{L L}$ are the corresponding partitions of the expanded admittance matrix. Therefore the relationship of bus voltages $V$ to generator voltages $E$ can be expressed as:

$$
V=-Y_{L L}^{-1} Y_{L G} E=R_{V} E
$$

\footnotetext{
${ }^{2}$ The mechanical power $P_{m_{i}}$ and machine inertia $H_{i}$ should not be confused with the error covariance $P$ and measurement Jacobian $H$ from the preceding section. While potentially confusing these are the variables used by popular convention in the respective fields.
}

where $R_{V}$ is defined as the bus reconstruction matrix. In a power flow model, the line flows are functions of bus voltages $V$, hence can be easily constructed as functions of generator voltages $E$ using (16).

The measurement model, which is discussed in [5] and [6], can be expressed in the form of equation (2) after linearization

$$
z=H x+v
$$

where $H$ is the corresponding Jacobian matrix, and $v$ is the measurement noise with normal probability distribution $p(v) \sim N(0, R)$. The measurement noise covariance $R$ can be determined experimentally, by off-line testing of the measurement devices over time and (if desired) under different conditions.

\section{B. The Kalman Filter}

We define $\hat{x}_{k}^{-} \in \mathcal{R}^{n}$ to be the a priori state estimate at time step $k$ given the knowledge of the process prior to $k$, so $e_{k}^{-} \equiv x_{k}-\hat{x}_{k}^{-}$is called the a priori estimate error and $P_{k}^{-} \equiv$ $E\left[e_{k}^{-} e_{k}^{-T}\right]$ is called the a priori estimate error covariance.

Similarly, We define $\hat{x}_{k} \in \mathcal{R}^{n}$ to be the a posteriori state estimate at time step $k$ given measurement $z_{k}$, so $e_{k} \equiv x_{k}-\hat{x}_{k}$ and $P_{k} \equiv E\left[e_{k} e_{k}^{T}\right]$ are called the a posteriori estimate error and the a posteriori estimate error covariance respectively.

The Kalman filter [11] estimates the state by minimizing the a posteriori estimate error covariance, in a recursive prediction-correction manner.

The prediction step is realized by the time update equations:

$$
\text { Prediction }\left\{\begin{array}{l}
\hat{x}_{k}^{-}=A \hat{x}_{k-1} \\
P_{k}^{-}=A P_{k-1} A^{T}+Q
\end{array}\right.
$$

The time update equations are responsible for projecting forward (in time) the previous state $x_{k-1}$ and error covariance estimates $P_{k-1}$ to obtain the a priori estimates for the next time step $k$. The correction step is carried out by a set of measurement update equations:

$$
\text { Correction }\left\{\begin{array}{l}
K_{k}=P_{k}^{-} H^{T}\left(H P_{k}^{-} H^{T}+R\right)^{-1} \\
\hat{x}_{k}=\hat{x}_{k}^{-}+K_{k}\left(z_{k}-H \hat{x}_{k}^{-}\right) \\
P_{k}=\left(I-K_{k} H\right) P_{k}^{-}
\end{array}\right.
$$

where $K$ is a $n \times m$ matrix called the Kalman gain matrix, $z_{k}$ is the actual measurement at time step $k, H \hat{x}_{k}^{-}$is the predicted measurement at time step $k$, and $\left(z_{k}-H \hat{x}_{k}^{-}\right)$is called the measurement residual or innovation.

$K$ reflects how we trust the actual measurement $z_{k}$ versus the predicted measurement $H \hat{x}_{k}^{-}$. From its expression, one can tell that larger values of $R$ place more weight on the predicted value while smaller values of $\mathrm{R}$ place more weight on the measured values. The measurement update equations are responsible for the feedback, i.e. for incorporating a new measurement into the a priori estimate to obtain an improved a posteriori estimate.

\section{Reduced Measurement-space Estimation}

As one would expect, performing dynamic state estimation with the KF/EKF (and in fact, any filter) can be a computationally intensive process. For the small system in 
[5], a regular PC was able to perform the computation fast enough for real time control applications. However there are three factors that increase the computational effort: the size of the system, the complexity of model components, and the number of measurements to be processed. In the "Correction" phase corresponding to equation (19), we have noticed the expensive cost of calculating the Kalman gain $K_{k}$. Because it involves the inversion of an $m \times m$ matrix $\left(H P_{k}^{-} H^{T}+R\right)$, with complexity of $O\left(\mathrm{~m}^{3}\right)$. This is a potential computation bottleneck when the number of measurements $m$ is too large, which unfortunately, is true for the modern power systems.

On the other hand, if we could reduce the measurement space dimension, i.e., only use a subset of measurements in each Kalman filter cycle to update (perhaps a subset of) the states, the computation can be sped up dramatically, according to SCAAT [9]. Now the question boils down to deciding which measurement subset to use at each cycle. Should we take the naive random approach? Or is there a much wiser method to help? We set our sights on the state space. Via principal component analysis (PCA) of the error covariance, we can "pin down" the subspace with the largest estimation uncertainty, i.e. the subspace most in need of corresponding measurements.

Due to the fact that covariance matrices are always symmetric and positive semidefinite, there are several important properties about them. Before the measurement update ("Correction" phase (19)) begins, let us consider the PCA of the $a$ priori error covariance matrix $P^{-}=U \cdot D \cdot U^{T}$ :

1) There exists an orthonormal basis $U\left(U U^{T}=U^{T} U=\right.$ $I$ where $I$ is the identity matrix), whose columns are the eigenvectors of $P^{-}$, such that the error covariance matrix expressed in this basis is diagonal. The axes of this new basis are called the Principal Components of $P^{-}$.

2) As the off-diagonal elements of this new diagonal covariance matrix $D$ are 0 , the new variables defined by this new basis (the projections of the a priori estimate error $e^{-}=x-\hat{x}^{-}$on the Principal Components) are uncorrelated.

3) The diagonal elements of this new matrix $D$ are the eigenvalues of $P^{-}$. So the variances of the projections of error $e^{-}$on the Principal Components are equal to the corresponding eigenvalues of $P^{-}$.

4) The eigenvalues in $D$ are ordered decreasingly. The $m^{\text {th }}$ eigenvalue corresponds to the $m^{\text {th }}$ eigenvector.

The principal components that correspond to the largest elements of $D$, indicate the axes in the state space that have the largest estimation uncertainties. We prefer to reduce these uncertainties first, thus need to find the subset of measurements that reduce the uncertainties most. This makes intuitive sense in any type of state estimation process. For example, in a 3D tracking application, if we use several cameras to estimate the location of a certain object, and we noticed the uncertainty is growing rapidly in one direction, then in the next cycle of filtering we would ideally use a camera which is looking in an orthogonal direction.

\section{Measurement Selection Procedure}

Similar to SCAAT, ReMeDySE also constrains the unknowns over time and refines the estimation continually, rather than waiting for a complete collection of observations to form. Nonetheless, it is the measurement selection that differs ReMeDySE from SCAAT. In ReMeDySE, we select the measurements that benefit our estimate the most (i.e. reduce estimation uncertainty most effectively) during each iteration.

After the "Prediction" phase (18) of each Kalman filter iteration, we have the $n \times n$ a priori error covariance matrix

$$
P^{-}=U \cdot D \cdot U^{T}
$$

where $D$ is the diagonal matrix consisting of the eigenvalues of $P^{-}$in decreasing order, and $U$ is the orthonormal basis whose columns are the corresponding eigenvectors. Notice that the full PCA can also be a quite time consuming process, especially if the state space is large. For this reason, in this paper we only investigate the first eigenvector $u_{1}$ in $U$, which represents the directions that we are most uncertain about in the state space. The largest eigenvalue and the corresponding eigenvector $u_{1}$ can be conveniently obtained by existing algorithms such as the power method.

Now consider the measurements. We can rewrite the measurement equation as

$$
z=H x+v=H U U^{T} x+v=(H U) x^{\prime}+v=H^{\prime} x^{\prime}+v
$$

Where $x^{\prime}=U^{T} x$ is the new state vector defined by the new basis $U, H^{\prime}$ is the corresponding $m \times n$ new measurement Jacobian matrix, and $v$ is the unchanged measurement noise vector with $p(v) \sim N(0, R)$. Here we assume $R$ to be an $m \times m$ diagonal covariance matrix, i.e. the measurement noise sources are uncorrelated.

Because the basis $U$ are composed of unit vectors, $H_{i j}^{\prime}$ can also be considered as the magnitude of the projection (i.e. the scalar projection) of the $i^{\text {th }}$ measurement direction vector in the direction of the $j^{\text {th }}$ basis in $U$. Notice that we now have

$$
H u_{1}=\left[\begin{array}{lll}
H_{11}^{\prime} & H_{21}^{\prime} & H_{31}^{\prime} \ldots H_{m 1}^{\prime}
\end{array}\right]^{T} .
$$

Intuitively, for the same basis, say $u_{1}$, the larger $H_{i 1}^{\prime}$ is, the better the corresponding $i^{\text {th }}$ measurement could reduce the uncertainty in this basis direction. However we should also keep in mind that different measurements have different amounts of noise. Thus for $u_{1}$ we create a ranking vector $r_{1}$ from $H^{\prime}$ and $R$ using the following adjustment:

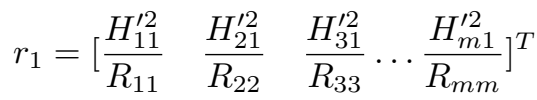

where $R$ is the measurement noise covariance matrix. The $m \times 1$ vector $r_{1}$ provides a means to evaluate the expected effectiveness of each measurement by scaling the corresponding Jacobian elements by the corresponding expected measurement noise levels. Now we are going to prove the following:

Lemma 1: The larger $\frac{H_{i 1}^{\prime 2}}{R_{i i}}$ is, the more effectively the $i^{\text {th }}$ measurement can reduce the uncertainty along direction $u_{1}$. 
Proof: It has been shown in [14] that the error covariance update in the "Correction" phase (19)

$$
\begin{aligned}
P & =(I-K H) P^{-} \\
& =\left(I-P^{-} H^{T}\left(H P^{-} H^{T}+R\right)^{-1} H\right) P^{-} \\
& =P^{-}-P^{-} H^{T}\left(H P^{-} H^{T}+R\right)^{-1} H P^{-}
\end{aligned}
$$

is equivalent to

$$
P^{-1}=\left(P^{-}\right)^{-1}+H^{T} R^{-1} H
$$

The inverse of the error covariance, $P^{-1}$, is often called the information matrix. According to (20), we have

$$
\begin{aligned}
P^{-1} & =\left(U D U^{T}\right)^{-1}+H^{T} R^{-1} H \\
& =U^{-T} D^{-1} U^{-1}+U^{-T} U^{T} H^{T} R^{-1} H U U^{-1} \\
& =U^{-T}\left[D^{-1}+(H U)^{T} R^{-1}(H U)\right] U^{-1} \\
& =U^{-T}\left(D^{-1}+H^{\prime T} R^{-1} H^{\prime}\right) U^{-1}
\end{aligned}
$$

Let us denote the matrix $\left(D^{-1}+H^{\prime T} R^{-1} H^{\prime}\right)$ in equation (26) by $\Sigma$, then $\Sigma_{11}$ is the "information" we now have regarding the new state variable in the direction $u_{1}$, which we were most uncertain about. This information value is increased/improved from $D_{11}^{-1}$ to $\Sigma_{11}$ by

$$
\left(H u_{1}\right)^{T} R^{-1}\left(H u_{1}\right)=\sum_{i=1}^{m} \frac{H_{i 1}^{\prime 2}}{R_{i i}}
$$

So if we are only willing to incorporate $m_{\sigma}$ measurements (instead of the full $m$ measurements) into the measurement update equation, the ones with the largest $H_{i 1}^{\prime 2} / R_{i i}$ ratios would be our choice.

We are able to easily locate the $m_{\sigma}$ measurements with the largest values among these $m$ elements in vector $r_{1}$ (23). Thus when our budget of computation time and memory space is tight, we could choose to use only $m_{\sigma}$ measurements in the next step but still achieve stable estimation results. Using this approach to reduce state estimation uncertainty is similar to fighting the Hydra in Greek mythology: if we are not able to destroy all the "heads" at once, at least we can aim at and cut off the most threatening "head" during each round.

\section{E. ReMeDySE Structure}

With modern shared-memory multi-core high performance computers, we can parallelize the algorithm to improve the computational efficiency of ReMeDySE in large-scale system dynamic state estimations.

Consider the power system dynamic state estimation model described in III-A, with a large measurement space. ReMeDySE utilizes the LoDiM algorithm [10] to have its main dynamic state estimation process running in the foreground; while the auxiliary measurement selection process as described in subsection III-D runs in the background. The ReMeDySE process can be expressed by the flow chart in Figure 1.
KF/EKF (foreground)

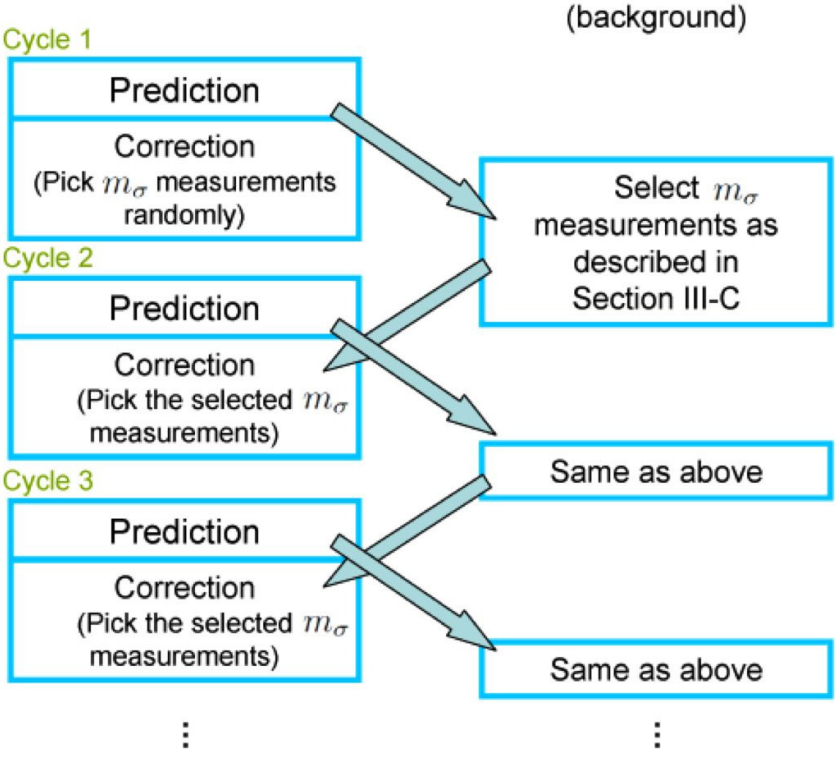

Fig. 1. The ReMeDySE flow chart

\section{Simulation Results}

We used the ReMeDySE approach on the standard 16generator 68-bus model representing the New England/New York interconnected system. We simulated a three-phase fault at bus 29, starting from $t=1.1$ and lasting for 0.05 second, as the dynamic event, representing a large disturbance emergency.

We simulated the dynamic state variables (the rotor angles and speeds of each generator) and recorded them as the actual dynamic states. The measurement set consisted of simulated PMU measurements: voltages and phase angles, combined with $1 \%$ random noise. In this paper, we made the strong assumption that all bus voltages and phase angles are measured by PMUs. In the future we will relax this assumption.

Fig. 2 depicts the actual and the estimated speed (using ReMeDySE with only 20 PMU measurements per cycle, and regular EKF with all PMU measurements per cycle) of generator 2 within 10 seconds from $t=0$ to $t=10$, to capture its reaction to this large disturbance. The estimate resolution of ReMeDySE is 0.01 second; while the estimate resolution of regular EKF is 0.2 second, due to the much larger measurement set involved in the computation. The detailed time complexities will be discussed in our future work.

For a better comparison. Fig. 3 zooms in the green dashedline window, and gives us a close-up look of the short window from $t=2$ to $t=5$ of our simulation. Similarly, Fig. 4 shows the rotor angle estimation results at this same generator during the same window. Generator 10 offers another example. Fig. 5 and Fig. 6 illustrate the rotor speed and angle estimation results at generator 10 respectively.

These plots demonstrate that under the experimental conditions, our proposed ReMeDySE approach outperforms the traditional EKF approach with higher accuracy. The primary reason is that with the standard batch approach to EKF-based dynamic state estimation, the entire measurement set is used as input, resulting in a lower estimation rate and higher latency. 


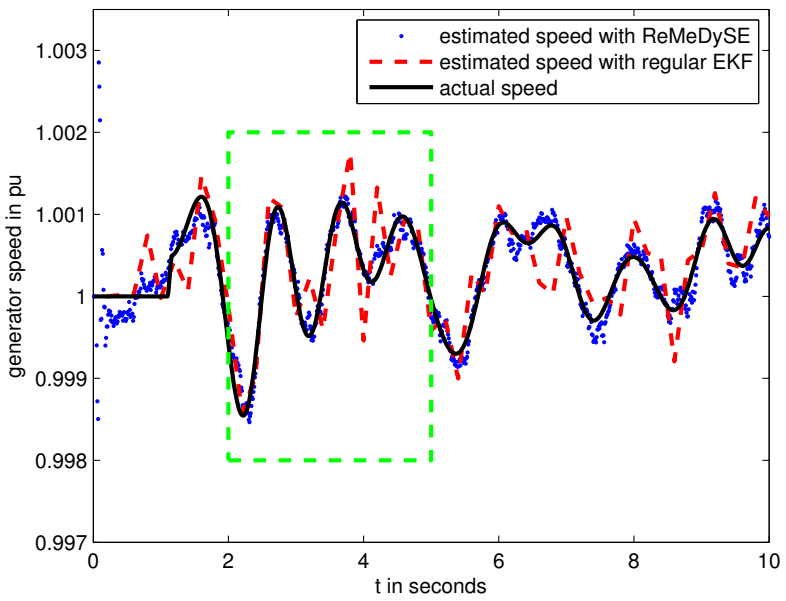

Fig. 2. Estimation of rotor speed at generator 2 using ReMeDySE and regular EKF, with a 3-phase fault at bus 29

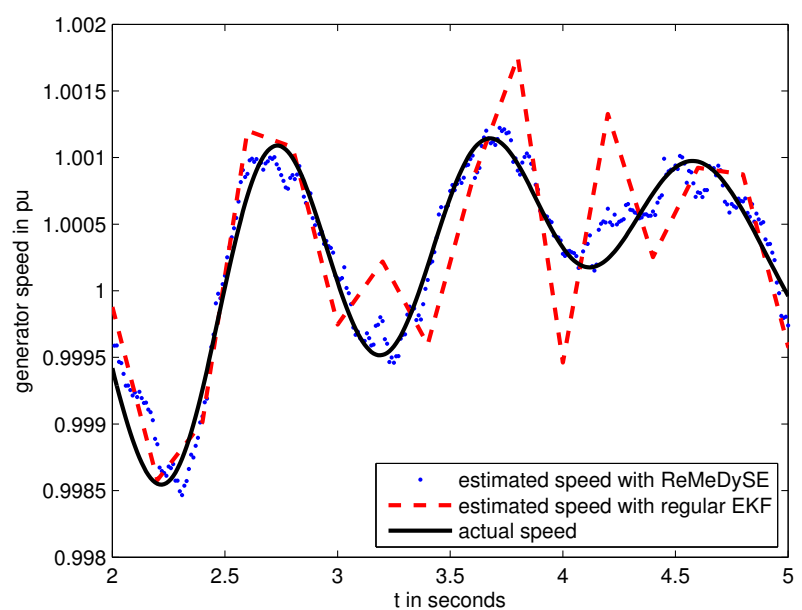

Fig. 3. The performance of ReMeDySE comparing to the regular EFK, during the short period shown in the dotted-line box from Fig. 2

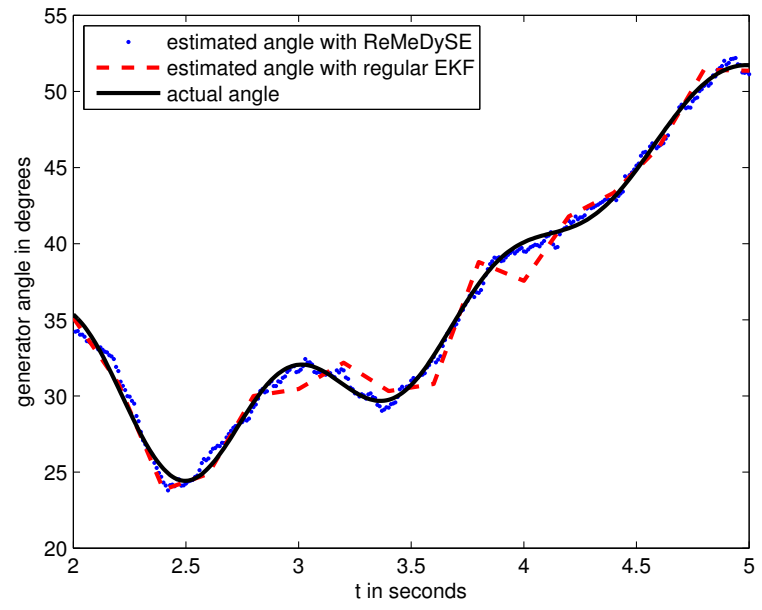

Fig. 4. Estimation of rotor angle at generator 2 using ReMeDySE and regular EKF

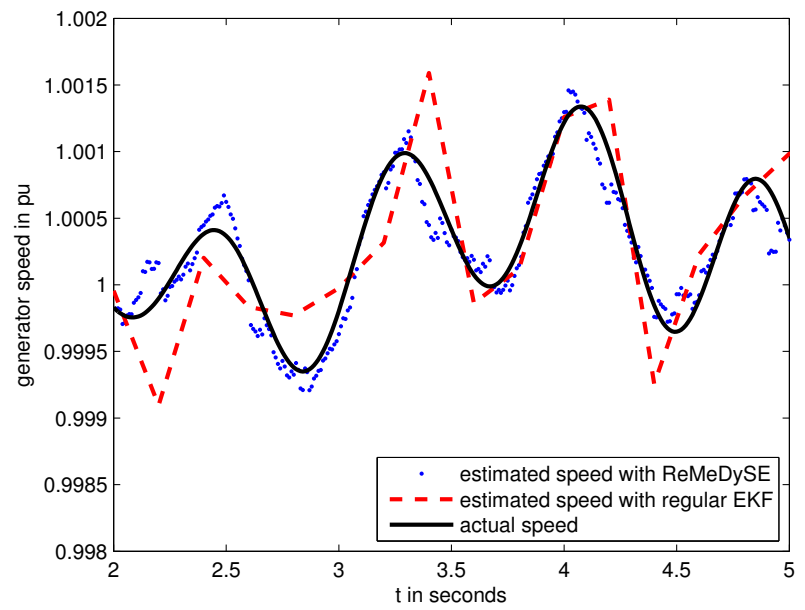

Fig. 5. Estimation of rotor speed at generator 10 using ReMeDySE and regular EKF

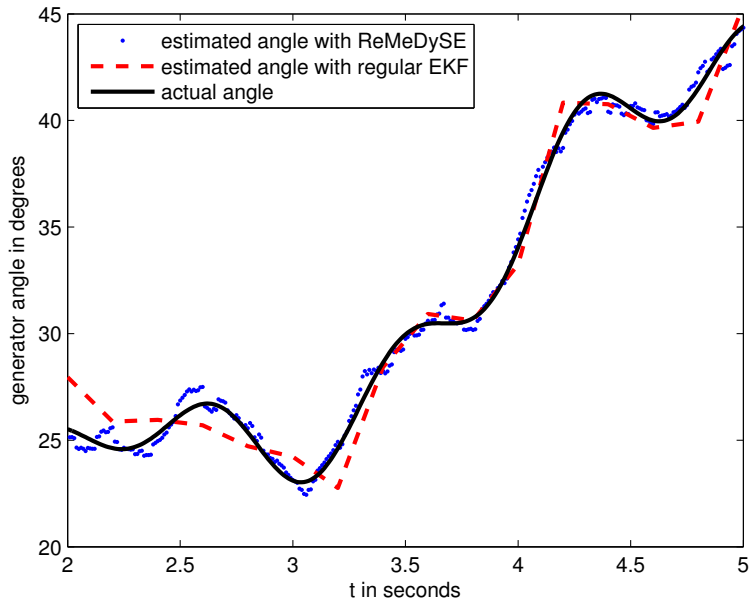

Fig. 6. Estimation of rotor angle at generator 10 using ReMeDySE and regular EKF

The dynamic nature of the state variables introduces additional noise (uncertainty) into the process, and this noise increases as the latency increases. Typically when latency is small, the measurement device noise dominates; when latency is large, the process noise dominates. In ReMeDySE, only a small, dynamically selected subset of the measurements is used. The result is a faster estimation cycle, combined with high accuracy PMU measurements, making it possible to achieve better dynamic state estimations under normal conditions.

\section{CONCLUSIONS}

In this paper we have proposed ReMeDySE, the Reduced Measurement-space Dynamic State Estimation method, an optimal measurement selection approach that can be applied to the traditional Kalman filter for dynamic state estimation problems such as power systems, where the measurement spaces are exceptionally large. The parallelized dynamic measurement selection procedure incorporates only the most critical measurement subspace into the computation, resulting in 
higher reporting rate and lower latency. Together with high frequency and accuracy PMU data, ReMeDySE can provide better estimation of large-scale dynamic state spaces such as power systems.

The PMU data in our simulations are ideal; while in practice, they can suffer from corruption and loss beyond noise. In the future we plan to explore methods for robust measurement selection based on statistical likelihood. Also, selecting certain measurement subspaces inherently delays the selection of the entire (remaining) measurement space, which could potentially delay the observance of an important state change. We plan to investigate the addition of measurement prioritization based on factors such as unexpected (deviating from prediction) state changes throughout the entire state space.

\section{ACKNOWLEDGEMENTS}

At the Pacific Northwest National Laboratory we acknowledge Ning Zhou, Pengwei Du, and Ruisheng Diao for helpful background discussions. This work was supported by a U.S. Department of Energy (grant DE-SC0002271) "Advanced Kalman Filter for Real-Time Responsiveness in Complex Systems," PIs Zhenyu Huang at PNNL and Greg Welch at UNC. At DOE we acknowledge Sandy Landsberg, Program Manager for Applied Mathematics Research; Office of Advanced Scientific Computing Research; DOE Office of Science. This work was also supported in part by the Office of Naval Research (award N00014-09-1-0813, “3D Display and Capture of Humans for Live-Virtual Training." At ONR we acknowledge Dr. Roy Stripling, Program Manager.

\section{REFERENCES}

[1] Atif S. Debs, Robert E. Larson "A Dynamic Estimation for Tracking the State of a Power System”. IEEE Transactions on Power Apparatus and Systems, vol. PAS-89, no. 7, September/October, 1970.

[2] Amit Jain, Shivakumar N. R. "Power System Tracking and Dynamic State Estimation” IEEE PES Power Systems Conference Exposition (PSCE) 2009.

[3] A. Phadke, J. Thorp, and M. Adamiak, "A new measurement technique for tracking voltage phasors, local system frequency, and rate of change of frequency" Power Apparatus and Systems, IEEE Transactions on, vol. PAS-102, no. 5, pp. 1025 C1038, may 1983.

[4] A. G. Phadke, J. S. Thorp, and K. J. Karimi, "State estimlatjon with phasor measurements" Power Systems, IEEE Transactions on, vol. 1, no. 1, pp. 233 C238, feb. 1986.

[5] Zhenyu Huang, Kevin Schneider, and Jarek Nieplocha "Feasibility Studies of Applying Kalman Filter Techniques to Power System Dynamic State Estimation" The 8th International Power Engineering Conference C IPEC2007 3-6 December 2007, Singapore

[6] Jinghe Zhang, Greg Welch, Gary Bishop, and Zhenyu Huang. “ Optimal PMU Placement Evaluation for Power System Dynamic State Estimation”. IEEE PES Conference on Innovative Smart Grid Technologies Europe (ISGT 2010), Chalmers Lindholmen, G?teborg, Sweden, October 10C13, 2010.

[7] Barbara Chapman, Gabriele Jost, and Ruud van der Pas, "Using OpenMP: Portable Shared Memory Parallel Programming.” the MIT Press, 2007

[8] Jarek Nieplocha, Bruce Palmer, Manojkumar Krishnan, and P. Sadayappan, " Overview of the global arrays parallel software development toolkit”. 2006 ACM/IEEE conference on Supercomputing, Tampa, Florida, 2006.

[9] Greg Welch and Gary Bishop, "SCAAT: Incremental Tracking with Incomplete Information”. Computer Graphics. T. Whitted. Los Angeles, CA, USA (August 3 - 8), ACM Press, Addison-Wesley: 333-344. 1997.

[10] Jinghe Zhang, Greg Welch, and Gary Bishop, "Power System State Estimation with Dynamic Optimal Measurement Selection”. To appear in Proceedings of 2011 IEEE Symposium on Com- putational Intelligence Applications in Smart Grid, (Paris, France), 11C15 April 2011.
[11] R. E. Kalman "A new approach to linear filtering and prediction problems". Transaction of the ASMEłJournal of Basic Engineering, 82(Series D):35C45, 1960.

[12] M.J. Goris, D.A. Gray and I.M.Y. Mareels "Reducing the computational load of a Kalman filter”. Electronic Letters Vol. 33 No. 181539 -1541, 28th August 1997.

[13] Shoujun Bian, Michael A. Henson "Measurement selection for on-line estimation of nonlinearwave models for high purity distillation columns". Chemical Engineering Science 61 (2006) 3210 C 3222

[14] Frank L. Lewis "Optimal Estimation with an Introduction to Stochastic control theory”. John Wiley and Sons, Inc. 1986.

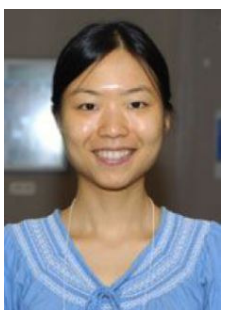

Jinghe Zhang is a graduate student in computer science at the University of North Carolina (UNC) at Chapel Hill. Her current research interests include optimal sensor placement for the power grid, and large scale estimation in general. Zhang has a B.S. from the University of Science and Technology of China (2006), and an M.S. from the University of Idaho (2008), both in mathematics.

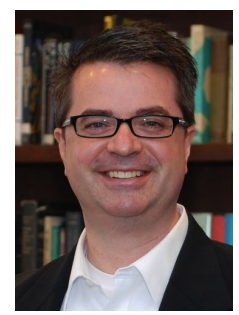

Greg Welch is a research associate professor of computer science at the University of North Carolina (UNC) at Chapel Hill. His primary research areas include stochastic estimation, virtual and augmented reality, human tracking systems, and 3D telepresence. Welch has a B.S.E.T. from Purdue University (1986), and a Ph.D. in computer science from UNCChapel Hill (1995). He is a member of the IEEE Computer Society and the ACM.

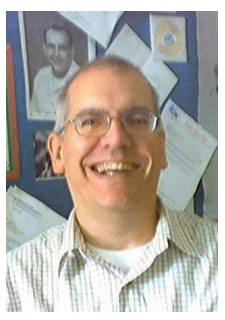

Gary Bishop is a professor of computer science at the University of North Carolina (UNC) at Chapel Hill. His current research interests include applications of computer technology to address the needs of people with disabilities, and systems for manmachine interaction. Bishop has a B.S.E.E.T. from Southern Technical Institute (1976) and a Ph.D. in computer science from UNC-Chapel Hill (1984).

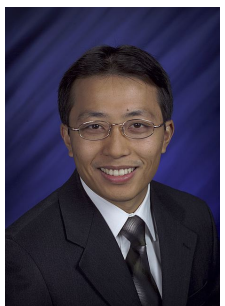

Zhenyu Huang is a staff research engineer at the Pacific Northwest National Laboratory, Richland, WA, and a licensed professional engineer in the state of Washington. His research interests include power system stability and control, high-performance computing applications, and power system signal processing. Huang (M'01, SM'05) received his B. Eng. from Huazhong University of Science and Technology (1994) and Ph.D. from Tsinghua University (1999). 